\title{
LA DIFICIL TAREA DE REFORMAR LA INSTITUCIONALIDAD REGULATORIA*
}

THE HARD TASK OF REFORMING REGULATORY INSTITUTIONS

\section{EDUARDO SAAVEDRA**}

ILADES-Universidad Alberto Hurtado

\begin{abstract}
Based on the book tribute to the Nobel Prize in Economics 2009 Oliver Williamson, this essay summarizes the fourteen chapters of the book and presents valuable applications to different issues of regulatory policy in Chile. Concludes this essay with a strong recommendation to policy makers and the applied academia to keep in mind a number of issues delivered by the new institutional economics, in particular those regarding reforms in the areas of utilities and public infrastructure.
\end{abstract}

\begin{abstract}
Resumen
Este ensayo toma el libro editado por Ménard y Ghertman, homenaje al Premio Nobel de Economía 2009 Oliver Williamson; hace una breve reseña de él, resume los catorce artículos que le componen y menciona valiosas aplicaciones de cada uno de ellos a diferentes tópicos de política regulatoria de servicios básicos en Chile. Concluye este ensayo con una fuerte recomendación a policy makers y académicos aplicados para tener presente una serie de elementos que entrega la nueva economía institucional al proponer reformas en sectores de servicios básicos e infraestructura.
\end{abstract}

* Reseña y aplicaciones para Chile de Regulation, Deregulation, Reregulation: Institutional Perspectives, editado por Claude Ménard y Michel Ghertman, Edward Elgar Publishing, 2009.

** Facultad de Economía y Negocios. Dirección: Erasmo Escala 1835, Santiago, Chile. Teléfono: (562)8897354. Fax (562)6920303. E-mail: saavedra@uahurtado.cl 


\section{INTRODUCCION}

En octubre de 2009 la Academia de Ciencias de Suecia otorgó el Premio Nobel de Economía al profesor Oliver Williamson, de la Universidad de California en Berkeley, una decisión bastante esperada en círculos académicos por los significativos aportes de este economista norteamericano al análisis de los fenómenos económicos de la gobernanza y los límites de la firma. ${ }^{1}$ El libro editado por los profesores Claude Ménard del Centro de Economía de la Sorbonne de la Universidad de París y Michel Ghertman de la Universidad de Niza, constituye un reconocimiento premonitorio al profesor Oliver Williamson y todo su trabajo en la economía institucional. Este libro fue publicado en los meses previos al anuncio del galardón del premio Nobel para Williamson.

Este ensayo está organizado, además de esta introducción, en cinco secciones. La primera es la reseña propiamente tal, las tres que siguen están relacionadas a las tres partes del libro: aspectos conceptuales, gobernanza e incentivos y procesos de desregulación. Termina el ensayo con una síntesis o epílogo. En cada una de las tres secciones ligadas a las tres partes del libro se resume capítulo a capítulo y se entrega una breve discusión de un caso aplicado a temas regulatorios en Chile en letra cursiva.

\section{RESEÑA}

Me parece que este libro es de enorme utilidad para académicos aplicados a temas de políticas públicas, en particular y dado los alcances del trabajo de Williamson y sus seguidores desde los años 70, a los temas de regulación y competencia en industrias con poder de mercado, léase servicios básicos e infraestructura pública, preferentemente. Con la lectura de este libro, los policy makers que trabajan en esas áreas pueden encontrar sustento, y uno que otro "tirón de orejas" a su trabajo aplicado. En otras palabras, este libro editado por Ménard y Ghertman a fines del año 2009 nos pone al día en una literatura tremendamente influyente en el día a día de muchos economistas, con aplicaciones a los temas de gobernanza y el desempeño en industrias como la eléctrica o las telecomunicaciones, entre otros.

Los diversos aportes de los autores de cada capítulo del libro, que revisaremos en las secciones siguientes, no sólo se quedan en aquellos elementos que constituirían un resumen histórico de la literatura, sino que ubicados a fines del primer decenio del siglo XXI buscan respuestas a nuevas preguntas que surgen del análisis de políticas públicas. El punto en común entre todos estos trabajos es precisamente lo que conocemos como economía de los costos de transacción, o más ampliamente hablando la nueva economía institucional.

1 Ver el comunicado de prensa en http://nobelprize.org/nobel_prizes/economics/laureates/2009/illpres. html (revisado el 25-may-2010). Como el mismo Williamson lo ha destacado en varios de sus trabajos, se entiende gobernanza (governance en inglés) como los medios o mecanismos a través de los cuales se pueden impartir órdenes, mitigar conflictos y obtener ganancias mutuas del intercambio entre agentes económicos. 
Los aspectos regulatorios en industrias con poder de mercado han sido desde los años 80 los objetos de estudio favoritos de esta literatura, no sólo por su importancia en el bienestar económico y social de la población, sino porque en particular los servicios básicos han mostrado una evolución enorme no sólo en los aspectos regulatorios sino también en los aspectos relacionados con su propiedad. ${ }^{2}$ Fenómenos como la privatización de estos servicios, las concesiones de obras de infraestructura, la integración de mercados monopólicos con industrias en donde la competencia se hacía cada día más real, entre otros; requieren una mirada interdisciplinar, en particular sus consecuencias de política pública. Es en tal sentido que la nueva economía institucional -de la que Oliver Williamson es sin dudas uno de sus más influyentes pensadores junto a los también ganadores del premio Nobel Ronald Coase y Douglas North- es la herramienta analítica ideal para estudiar estos fenómenos.

\section{ASPECTOS CONCEPTUALES}

El libro se organiza en tres partes. La primera de ellas corresponde a la estructura analítica, esto es, la estructura conceptual requerida para comprender los aportes de Williamson, así como comprender el alcance de sus aplicaciones a los temas de política pública real. Cuatro capítulos constituyen esta parte, siendo la primera de ellas del propio Oliver Williamson.

El artículo de Williamson denominado "Transaction Cost Economics: The Precursors" hace una revisión bastante extensa y en perspectiva de las contribuciones básicas a esta disciplina que hicieron sus precursores: Hayek, Buchanan, North, Coase, entre varios otros. En este trabajo, Williamson pone en el ojo de la discusión metodológica de tópicos de políticas públicas la necesidad de tener siempre presente los elementos de gobernanza, en particular el rol de los contratos debido a los costos de transacción envueltos y sus consecuencias y factibilidad de soluciones. Al respecto, el autor hace una crítica profunda a acciones voluntaristas propias de reformadores con muy buenas intenciones, pero con débil arraigo en aspectos conceptuales, lo que al final indefectiblemente han conducido a experimentos fallidos en materia de desregulación de mercados potencialmente competitivos.

La liberalización del sector eléctrico en Chile en los años 80 es una muestra de lo poco cuidadoso que se puede ser en los procesos de desregulación cuando se supeditan objetivos dogmáticos a buenos diseños de la institucionalidad de esos mercados. La acelerada privatización de ENDESA, sin cuidado en el diseño regulatorio y en los vacíos legales que se dejaba, en particular los peajes eléctricos, y la integración vertical de esta empresa como dueña de los activos de transmisión, generó una serie de conflictos con otros generadores en los años 90. La solución finalmente fue la venta

2 Algunas referencias obligadas son los textos de Armstrong, Cowan y Vickers (1994) y Newbery (1999). Una síntesis de trabajos aplicados a América Latina puede encontrarse en, Paredes, Sánchez y Fernández (1995). 
de los activos de transmisión a un operador independiente (fines de los 90) y la modificación a la tarificación de los peajes de transmisión en el año 2003. La experiencia chilena mostró lo costoso de corregir los vacíos de la regulación toda vez que con la privatización se asignan derechos de propiedad que se ven afectados con cualquier modificación legal. Este caso nos enseña algo central en el diseño regulatorio, la secuencia liberalización-privatización importa, luego un cuidadoso diseño regulatorio es fundamental previo a desregular esa industria.

El profesor Gary Libecap, de la Universidad de California en Santa Bárbara, estudia los efectos distributivos de la asignación de los derechos de propiedad en su artículo "Property Rights Allocation of Common Pool Recourses". Los derechos de propiedad son centrales en la literatura de la nueva economía institucional, explicando de acuerdo a North gran parte de las asimetrías observadas en los patrones de desarrollo de los países. ${ }^{3}$ El trabajo de Libecap analiza tres diferentes mecanismos de asignación de los derechos de propiedad, a saber reglas de derechos históricos (o reglas de primer poseedor), reglas de asignación uniforme y las reglas de subasta, discutiendo en bastante detalle acerca de los pros y los contras de cada uno de ellos. El autor aplica esta discusión a tres áreas que son actualmente bastante atingentes, como la asignación de derechos de exploración y explotación de gas y petróleo bajo la superficie terrestre, los permisos de emisión de contaminación ambiental y los derechos de extracción pesquera.

Actualmente está en el tapete de la discusión pública la asignación de los derechos de pesca extractiva en Chile. Más allá de la duración de esos derechos, que si deben ser para siempre o limitados a un cierto número de años, un tema que debe ser zanjado por las autoridades competentes, es el modo en que estos debieran ser asignados. Algunos empresarios que representan a quienes actualmente tienen los derechos de propiedad argumentan la necesidad de que se reconozcan los derechos históricos de los empresarios, y por lo tanto y al igual que en el año 2001 cuando se usó esta regla para asignarles tales derechos por 10 años, esperan que ellos sean los favorecidos nuevamente, aunque esta vez parece ser del todo razonable la asignación indefinida de esos derechos. La ortodoxia económica nos hace pensar a otros que sería preferible una licitación de esos derechos, de forma que sea la competencia ex-ante o por la cancha el mejor mecanismo para asignar los derechos de pesca, en particular si se determinara que en esta oportunidad los derechos se debieran asignar indefinidamente. Con todo, el trabajo de Libecap nos muestra que no hay dogmas y las tres opciones que él menciona en su trabajo debieran ser estudiadas en sus propios méritos a la luz de este caso particular.

3 North (1990). Literatura adicional al respecto y que muestra la importancia de las instituciones y los derechos de propiedad en la calidad de la política pública y su subsecuente efecto sobre el desarrollo económico se encuentra en Lora (2001) y Kaufmann, Kraay y Mastuzzi (2009), por ejemplo. 
En el artículo "An Institucional Theory of Public Contracts: Regulatory Implications" Pablo Spiller, profesor de la Universidad de California en Berkeley, utiliza el enfoque de contratos para estudiar las principales diferencias entre los contratos privados versus los contratos firmados por el gobierno. Spiller va más allá de muchos trabajos que típicamente analizan los efectos en los contratos de la incapacidad del Estado para comprometerse creíblemente en el largo plazo, hecho que ciertamente es central en muchas reformas acometidas en materias regulatorias. Este autor muestra que unidas a la anterior hay incertidumbre adicional que genera incerteza regulatoria y que proviene del comportamiento oportunista de terceras partes, en otras palabras de grupos de interés a las que las políticas públicas le afectan indirectamente. Spiller muestra que la acción de estos grupos de interés termina por imponer rigideces a los contratos, lo que degrada la introducción de incentivos y en definitiva lleva a la implementación de modos ineficientes de organización de la actividad económica. El autor soporta sus predicciones teóricas con evidencia empírica para las concesiones de contratos en América Latina.

Luego de varios casos en que se pagaron importantes sumas de dinero a altos ejecutivos de empresas públicas en Chile (Correos de Chile en 2001 o CODELCO en 2006, por ejemplo), se escuchan voces para aplicar esquemas de incentivos en empresas públicas similares a los usados en el sector privado, tal que esos pagos aparezcan relacionados al desempeño de la empresa y no a todo evento. Las enseñanzas que nos entrega el trabajo de Spiller son una señal de cautela: debido a las idiosincrasias propias de las empresas públicas, los incentivos monetarios al desempeño en general no inducen a los ejecutivos públicos a actuar mancomunadamente con el objetivo esperado. Todo pareciera indicar que en lugar de diseñar pagos por incentivos poderosos para los gerentes de las empresas públicas, se requieren reformas a la institucionalidad de los gobiernos corporativos en esas empresas, como por el rol de los directorios, los objetivos de estos y su rol con los responsables de la gestión de las empresas públicas.

Steve Tadelis, profesor de la Universidad de California en Berkeley, también estudia los incentivos en el sector público comparado a esos en el sector privado. Utilizando como contrafactual las licitaciones competitivas en el sector privado, Tadelis muestra que el gobierno no puede negociar con las partes que participan de la licitación previamente a la adjudicación del contrato, como sí es regularmente hecho en licitaciones privadas, diferencia que es explicada en el artículo anterior de Spiller. Esto tiene como consecuencia que los contratos de adquisiciones del sector público operan con más rigideces y generan restricciones y costos adicionales a los agentes que participan de la licitación. Argumenta Tadelis que los contratos negociados pueden ser menos efectivos en seleccionar a los oferentes con menores costos, pero este tipo de prácticas imponen costos mayores a eventuales revisiones de contratos que sean necesarias ex-post.

Para apurar un profundo cambio al sistema de transporte público en Santiago y asegurar la participación de los empresarios privados, durante 
la administración del Presidente Lagos el Ministerio de Transportes firmó contratos muy mal diseñados con estos operadores en que se les aseguraba un pago por los kilómetros recorridos, ipero en donde no se monitoreaba que los buses estuvieran operando! Como manifiesta Tadelis, estos contratos no tenían cláusulas de rompimiento de contrato y por lo tanto el gobierno de la Presidenta Bachelet que implementó el sistema tuvo que realizar enormes esfuerzos para acordar la modificación de esos contratos. Ciertamente los contratos privados contienen cláusulas de salida, pero como ello no es así en los contratos públicos, los gobiernos debieran tener mucho más cuidado con qué se comprometen. El Transantiago es un claro ejemplo de cómo no se debe proceder.

En el capítulo desarrollado por Claude Ménard "From Technical Integrity to Institutional Coherence: Regulatory Challenges in the Water Sector" el autor nos ilumina acerca de la dificultad de implementar diversos modelos de organización con funciones técnicas que mantengan la integridad del sistema como un todo, dificultad que genera ineficiencias importantes en la producción, distribución y recolección de agua potable. Ménard argumenta que lo que él llama instituciones microeconómicas juegan un rol central en garantizar que las transacciones fundamentales sean monitoreadas dentro de una estructura coherente de análisis y acción. Las instituciones microeconómicas son aquellas que caen entre la entidad más administrativa encargada del despacho (comando y control) y el regulador sectorial.

A modo de ejemplo, en el sector eléctrico se tiene además del regulador sectorial, la Comisión Nacional de Energía en Chile, una serie de organismos, unos más técnicos que otros, que toman decisiones de control, monitoreo, despacho, fiscalización, etc. Entre otros, el Centro Económico de Despacho de Carga como organismo independiente y encargado de la operación del sistema, la Superintendencia de Electricidad y Combustibles como organismo encargado de la fiscalización de la calidad del servicio y el Panel de Expertos del sector eléctrico como entidad independiente encargada de resolver los conflictos entre empresas del sector en la interpretación de la normativa vigente. Otros organismos tienen una acción indirecta sobre el desempeño del sector eléctrico, como la Dirección de Aguas del Ministerio de Obras Públicas, el Ministerio del Medioambiente, la Empresa Nacional del Petróleo. Lo interesante es que estas instituciones microeconómicas en la jerga de Ménard no surgen desde un principio sino que varias de ellas van apareciendo o van perfeccionando su mandato en la medida que se desarrolla la institucionalidad en esta industria. 


\section{GOBERNANZA Y DESEMPEÑO}

La segunda parte del libro se refiere a cómo la gobernanza impacta el desempeño de diversos sectores que son sometidos a fuertes reformas económicas. Son cinco trabajos que dan vida a esta parte del libro.

En el primero de ellos, Luis Andrés, José Luis Guasch y Sebastián López, todos del Banco Mundial y Guasch además profesor de la Universidad de California en San Diego, estudian el impacto de la capacidad de las agencias regulatorias en el desempeño del sector eléctrico. Los autores muestran que la existencia, experiencia y capacidad de gobernanza de estas agencias regulatorias tienen un impacto significativo en el desempeño de los sistemas eléctricos en América Latina. Además de constatar empíricamente este hecho, mostrando que el diseño de instituciones sí importa, lo que de por sí es valioso desde la perspectiva de la política pública, es enormemente interesante de este trabajo la rica discusión metodológica que muestra la construcción del índice de gobernanza regulatoria creado y utilizado por estos autores.

Chile muestra una política energética que desde los años 80 se ha construido a partir de dos pilares fundamentales: seguridad energética y eficiencia económica. No obstante, sólo a fines del gobierno del Presidente Lagos se redactó explícitamente esta política en donde los elementos de eficiencia y sustentabilidad energética aparecen como necesarios de tener presente pero a un nivel claramente menos prioritario que los dos pilares fundamentales. Sin embargo, la llegada del gobierno de Bachelet en el 2006 y sus compromisos ambientales derivaron en que este objetivo fuese puesto por encima de los dos anteriores, modificándose la política energética de Chile por la vía, por ejemplo, de asegurar en el despacho un mínimo de energía proveniente de fuentes renovables no convencionales, como es el caso de la energía eólica. Esto gatilló una ola de inversiones en Chile, pero el hecho de que se trata de fuentes de energía más caras que otras disponibles, como el carbón, por ejemplo, elevó el costo medio de largo plazo y por lo tanto esto debiera llevar a una caída en el crecimiento potencial del país. Una muestra de que no basta con la experiencia y la capacidad de gobernanza de los gobiernos, sino que además influye en el desempeño del sector el cómo los objetivos políticos se imponen por sobre el racionamiento técnico, sin que esos cambios hayan incluso sido discutidos en el Congreso.

El artículo "Vertical Relations and 'Neutrality' in Broadband Communications" de Howard Shelanski, profesor de la Universidad de California en Berkeley, nos introduce en el siempre vigente tema de cómo regular el acceso de los proveedores de aplicaciones a las redes de telecomunicaciones. De hecho, para el autor no es claro que este tipo de acceso debiera necesariamente ser regulado. Esto por cuanto aunque es válido pensar que en la última milla existen suficientes elementos físicos que no justifican la proliferación de redes de acceso, lo que en consecuencia genera poder de mercado en los dueños de esas redes, no es claro si se deba obligar al inmediato acceso libre de todo pago a cualquier proveedor. El autor discute acerca de las posiciones en juego: neutralidad en las reglas de uso de las redes versus derechos discriminatorios 
que permiten a los dueños de las redes imponer restricciones a los proveedores en su uso. Shelanski no entrega una conclusión que uno esperaría con ansias, pues de acuerdo a él este tema no tiene una solución jurídica ni económica clara y sólo el tiempo y el desarrollo de nuevas tecnologías deberían entregar esta respuesta.

Para quien hace esta reseña es difícil entender la política de neutralidad en las telecomunicaciones, toda vez que se trata de una industria que no parte de cero, sino que son los cambios tecnológicos los que atraen nuevos negocios y nuevas formas de ofrecer servicios que en algunos casos ya existían, por ejemplo el de la telefonía. Las telecomunicaciones se basan en enormes inversiones que una vez hundidas requieren mantener reglas del juego claras que permitan su financiamiento; sin embargo, por ejemplo, la aparición de la telefonía IP, que se monta sobre la red existente pero no es claro o barato identificar su uso, genera el problema que si se siguiera una política de neutralidad de la tecnología, usaría las redes existentes sin haber pagado por ellas. Como consecuencia, no competiría neutralmente con el dueño de las redes que ofrece el servicio de telefonía fija. La respuesta del dueño de las redes es obvia, subinvierte y por lo tanto genera ineficiencia productiva. A todas luces, pareciera que una política de entrada abierta que sólo respete parcialmente el compromiso de recuperación de las inversiones puede afectar a los propios consumidores.

Magali Delmas de la Universidad de California en Los Angeles, María MontesSancho de la Universidad Carlos III, Michael Russo de la Universidad de Oregón y Yesim Tokat de la consultora Bernstein Global Wealth Management, en su artículo "Desregulación, Eficiencia y Desempeño Ambiental: Evidencia desde la Industria Eléctrica" comparan los efectos en la eficiencia económica y en el medio ambiente de los procesos de desregulación del sector eléctrico en Estados Unidos. Ellos muestran que la desregulación llevó a importantes reingenierías de activos dentro de las empresas, lo que originó nuevas estructuras organizacionales y derivó en impactos negativos sobre la eficiencia económica global, en particular por el mayor poder de mercado generado, pero a importantes beneficios por el lado del uso de energías renovables. Este resultado, aparentemente contraintuitivo, muestra que los procesos desregulatorios son tremendamente complejos, razón por la cual argumentan los autores que no es posible arribar a resultados definitivos acerca de los impactos de largo plazo de estos procesos desregulatorios, a no ser que se contara con nueva y más robusta información al nivel micro que sirviera específicamente para tales fines. En otras palabras, la evidencia empírica no es capaz, con la información disponible, de responder a la pregunta acerca de si la desregulación o liberalización de los mercados es beneficiosa o perjudicial a los intereses de largo plazo de la sociedad.

Es interesante lo que sucedió en Chile con la privatización de los servicios sanitarios y su impacto ambiental. Debido a que el Estado carecía de los recursos para invertir en costosas plantas de tratamientos de aguas servidas, este problema ambiental y sanitario era de importancia en las grandes ciudades del país. La llegada de recursos frescos de manos del sector privado, y la imposición de realizar estas inversiones cuando las empresas se privatizaron, 
permitió que la calidad de las aguas para el regadío mejorara ostensiblemente. No obstante, esto ha traído aparejado un mayor costo del servicio de provisión de agua potable y alcantarillado, pues esas inversiones deben ser pagadas. El dilema no es que se paguen esas inversiones, pues pasaron el test de la evaluación social, sino que por ser hechas por empresas privadas se generan rentas informacionales que tienen como contraparte ineficiencia en asignación de recursos. ¿Cuán grandes son esas rentas comparadas a los beneficios de las inversiones en plantas de tratamiento de aguas? Me parece que es una pregunta empírica no respondida en Chile e interesante a la luz del artículo de Delmas y sus coautores.

El capítulo 9 del libro es "The Achievement of Electricity Competitive Reforms: A Governance Structure Problem?" de Jean-Michel Glachant, profesor de la Universidad Europea de Florencia, y Yannick Perez, investigadora de la Universidad de París, realizan un análisis comparado de dos posibles reformas a la operación del sistema de transmisión eléctrica de Europa, el del operador del sistema independiente (ISO por sus siglas en inglés) con el del operador del sistema de transmisión (TSO en inglés). Los autores argumentan que la complejidad de los sistemas de transmisión eléctrica es tal que cualquiera sea el modelo de gobernanza que se implemente en esta industria, se requiere en ambos que esta implementación sea realizada en forma secuencial; esto es, partiendo por bloques menos interdependientes y avanzando de esa forma hacia un sistema más complejo. Lamentablemente los autores no son capaces, por falta de información, de concluir si ya sea el modelo norteamericano (ISO) o el europeo por tradición (TSO) es el más eficiente para ser implementado en una red de transmisión integrada en todo el continente.

En el año 2000 la Comisión Nacional de Energía propuso una reforma profunda a la ley eléctrica en Chile, que buscaba hacerse cargo de los problemas con la tarificación y las inversiones en la transmisión eléctrica, fuente de numerosos y profundos conflictos en los años 90, pero además proponía cambiar el sistema de despacho desde uno de centralizado o despacho por mérito (a costo marginal) por uno descentralizado o de subastas en una bolsa de energía. Sobre lo último, se buscaba reducir las barreras a la entrada al mercado mayorista, la falta de transparencia en su operación y la incompletitud del Mercado spot y el desarrollo de un mercado minorista competitivo al cual accedan todos los agentes. Finalmente, el exceso de ambición en la reforma y, sobre todo, el desconocimiento de una evaluación seria que mostrara que el cambio era socialmente beneficioso, llevó a una reforma acotada o "ley corta" en el año 2003 sólo en el tema de tarifas de transmisión. Aún está pendiente la reforma larga, en particular en abrir el mercado de comercialización de energía.

Richard Geddes, profesor de la Universidad de Cornell, nos muestra enseguida el caso de la empresa estatal de correos de Estados Unidos en su artículo "The US Postal Service". A pesar de que esa empresa es una joya para los estándares latinoamericanos, no lo es en su país pues la evidencia es clara al compararla con un par de operadores privados, como Fedex y UPS. Muestra Geddes que a pesar de ser objetivo este pobre 
desempeño de US Postal Service, ha existido una enorme resistencia a su modernización, en particular a la enajenación de activos en segmentos de su mercado en que operan competidores privados con mayor eficiencia. Ello ha generado lo que en Chile conocemos como una economía mixta, o en jerga de la nueva economía institucional nos encontraríamos con US Postal Service como una organización híbrida (Ménard, 2006). El autor entrega diversas explicaciones a lo que él considera una liberalización excesivamente lenta, siendo cruciales pero no las únicas la existencia de grupos de interés que se oponen a estos cambios y el propio comportamiento oportunista del gobierno en bloquearlos.

Acordando con Geddes que muchas de las reformas a empresas públicas encuentran su principal escollo en sus propias gerencias, empleados o en sus grupos de interés (públicos y privados), la evidencia teórica y empírica nos muestra que las economías mixtas tienen sus pros y sus contras. ${ }^{4}$ Por ejemplo en Chile hay quienes abogan por una enajenación de activos en ENAP, Banco del Estado o el mismo Correos de Chile, aduciendo que en varios de sus segmentos de mercado operan empresas privadas. Las ventajas que tienen las empresas públicas que operan en mercados oligopólicos es que ellas reducen el poder de mercado o inhiben la formación de carteles (Banco del Estado y el pass through en las rebajas de tasas de interés), en mercados con fuerte incertidumbre o ries go no medible es que ellas facilitan las inversiones que no son acometidas por el sector privado (ENAP y la planta regasificadora de gas natural), o en mercados con demanda insuficiente es que ellas permiten la cobertura de servicios a toda la sociedad (oficinas de Correos en zonas rurales).

\section{PROCESOS DE DESREGULACION}

La tercera y última parte del libro estudia algunas complejidades en el movimiento hacia una mayor liberalización de industrias y cómo es posible observar en la práctica incluso movimientos de desregulación (vuelta atrás o hacia una mayor regulación). Esta parte consta de cinco capítulos, donde nuevamente la atención recae en electricidad y telecomunicaciones, con un interesante análisis de la industria financiera.

En efecto, el artículo "The Sarbanes-Oxley Act at a Croassroads" escrito por la profesora de la Universidad de Yale Roberta Romano nos muestra cómo los problemas de implementación de la ley Sarbanes-Oxley ha llevado a la imposición de mayores controles a la industria financiera, lo que ha derivado en la creación de imperfecciones en el funcionamiento del mercado de capitales y, consecuentemente, mayores costos de fondos que crean más barreras para el crecimiento en particular en las empresas de

4 Diversos trade off aparecen en mercados oligopólicos o incompletos, siendo la propiedad estatal en paralelo a las iniciativas privadas una opción que puede ser deseable desde el punto de vista incluso de la asignación de recursos (Cremer, Marchand y Thisse, 1989; De Fraja y Delbono, 1989). Este tipo de organizaciones existen ampliamente en América Latina, según muestra Fischer-Bollin (2008). 
menor tamaño. El problema es, por lo tanto, en cómo lograr que las modificaciones legales necesarias para dar mayor dinamismo y reducir el costo de fondo de las empresas sigan ese camino y no terminen entorpeciéndose por culpa de la presión de la media y los intereses cortoplacistas de los legisladores; o lo que Dixit (1996) llamaría costos de transacción políticos. La respuesta que da la literatura de la nueva economía institucional es que iniciativas pro mercado deberían evitar las modificaciones legales, otorgándole mayores poderes discrecionales al regulador sectorial (SEC).

La propuesta de Romano tiene sentido en el contexto de una reforma que buscando proteger el buen funcionamiento de la industria y evitar situaciones como las generadas por Enron, Worldcom y otras a fines de los 90, termina imponiéndole un freno al desarrollo. Sin embargo, en mi opinión, evitar el paso por el Congreso en toda reforma que por los mercados involucrados sea mediática tiene el riesgo más serio aún de debilitar en el largo plazo el desarrollo institucional de los países. Tal me parece que es el caso de las telecomunicaciones en Chile, cuya ley data de 1982 y sistemáticamente los diversos gobiernos han evitado realizar modificaciones, a pesar de que el dinamismo de la industria ha dejado obsoleta la ley en varias de sus partes. En particular, la ley no dice nada respecto de la metodología de cálculo de los cargos de acceso entre empresas móviles y entre móviles y fijas, tema prácticamente inexistente hace 30 años pero altamente relevantes desde hace unos 10 años al menos.

Los profesores Adam Fremeth, de la Universidad de Minnesota, y Guy Holburn, de la Universidad de Western Ontario nos muestran en su trabajo "Information Asymmetries and Regulatory Rate-Making: Case Study Evidence from Commonwealth Edison and Duke Energy Rate Reviews" acerca de la dificultad práctica de introducir mecanismos para reducir las asimetrías de información en los mercados eléctricos. Llama la atención esto dado el enorme avance en la literatura económica de los últimos 30 años respecto de la necesidad de imponer tarifas que dejen menos rentas de información a las empresas reguladas. Analizan los autores tres posibles vías para reducir estas asimetrías de información: i) conocimiento adquirido por el regulador en su experiencia pasada, ii) conocimiento de la firma basado en información que las propias empresas deben entregar estandarizadamente al regulador y iii) información provista por grupos de interés. Fremeth y Holburn aplican su trabajo a dos empresas del sector eléctrico y muestran que cuando grupos de interés organizados que velan por su propio interés son capaces de revelar información valiosa para una mejor regulación tarifaria.

La idea de los public hearings o procesos participativos en las decisiones regulatorias en Chile no es nueva, aplicándose en la legislación ambiental (Ley $N^{o} 19.300$ de bases generales del medio ambiente) y con impacto sobre las decisiones de inversión en plantas eléctricas, así como en los servicios básicos respecto principalmente de la fiscalización de la calidad del servicio prestado. No obstante, no existen instancias de participación ciudadana en los procesos de fijación tarifaria de los servicios básicos, los que de ser 
correctos los resultados de Fremeth y Holburn podrían incorporarse en las bases técnicas de los procesos tarifarios en sanitarias, distribución eléctrica y cargos de acceso en telecomunicaciones.

En el artículo "Adaptation in Long-Term Exchange Relations: Evidence from Electricity Markets Contracts" de Dean Williamson, de la Antitrust Division del Departamento de Justicia de Estados Unidos y de la consultora Economic Analysis Group, se introduce en el corazón de la naturaleza de la firma, el problema de hold up, el aporte fundamental que le valió a Oliver Williamson el Premio Nobel de Economía de acuerdo a la Academia sueca. Dean Williamson analiza más de 100 relaciones contractuales entre productores y comercializadores de aparatos de generación eléctrica, básicamente buscando cómo estos contratos eligen una combinación de cuatro herramientas que las empresas tienen a su disposición para hacer frente al problema de subinversión en activos específicos: duración de los contratos, derechos a veto de las partes, reglas de compartimiento de riesgos y el ratio de apalancamiento en cada firma (relación deuda-capital). ${ }^{5}$ A pesar de que la duración de los contratos ha sido la materia más largamente estudiada para explicar las diferentes estructuras de gobernanza que pueden elegirse de acuerdo al grado de especificidad de activos, la incertidumbre y la repetición de las transacciones, este trabajo muestra que en la práctica se elige una combinación de las cuatro herramientas que están a disposición de las empresas al momento de escribir el contrato.

La evidencia de contratos usados en la práctica también considera que la repetición de las relaciones contractuales lleva a la aparición de contratos relacionales; esto es, contratos más bien implícitos y que basan su cumplimiento en la reputación que se juegan las empresas. No existe evidencia para Chile al respecto, pues las empresas son muy celosas de sus contratos comerciales. Sin embargo, por las características de mayor concentración e integración vertical, el poco número de proveedores en las grandes industrias en el país, y una justicia poco preparada para resolver conflictos comerciales con certeza y prontitud se deberían esperar contratos más relacionales que aquellos estudiados para la industria eléctrica en Estados Unidos. ${ }^{6}$ Con todo, esto es materia de futura investigación aplicada.

En el capítulo 14 Jackie Krafft de la Universidad de Niza y Evens Salies del Instituto de Ciencias Políticas de Francia se adentran en las imperfecciones en el mercado de las telecomunicaciones con su trabajo "Why and How Should New Industries with High Consumer Switching Costs be Regulated? The Case of Broadband Internet in France". Los costos de cambiarse de proveedor son sin dudas uno de los temas más apasionantes en la actualidad no sólo en los servicios básicos, sino también en industrias tan importantes como las aerolíneas y la del retail, existiendo férreos defensores

5 Para el lector no experto en teoría de contratos e incentivos, se recomienda una mirada detallada al primer capítulo de Bolton y Dewatripont (2005).

6 Una discusión acerca de las relaciones contractuales y su evidencia se encuentra en Holmström y Roberts (1998) y Baker, Gibbons y Murphy (2002). 
de las prácticas de crear endógenamente costos de cambio, liderados por todos los graduados de las escuelas de negocios, y otros férreos opositores que encuentran en esto un mecanismo para ablandar la competencia en esos mercados, quién más que los economistas expertos en organización industrial. Los autores argumentan que la lenta penetración de las conexiones de redes en Francia se debe a los costos de cambio que experimentan los usuarios. Así, Krafft y Salies ven con cautela el casi dogma de la liberalización que dice "a mayor innovación tecnológica en una industria, mejor es desregularla y dejar que el mercado opere libremente y se autorregule". Los autores plantean como alternativa a una completa desregulación un rol más activo de las autoridades antimonopólicas y mantener algún grado de regulación para así romper las estructuras de dominancia que surgen en estas industrias con dueños/operadores de redes, y que los costos de cambio impiden que la competencia los elimine.

La portabilidad numérica ha sido uno de los caballitos de batalla de los entrantes en la industria de las telecomunicaciones en Chile, pues y consistente con el trabajo de Krafft y Salies, la carencia de esta portabilidad sería la explicación de por qué las compañías entrantes en telefonía fija después de 20 años de competencia nunca pudieron hacer frente a Telefónica. Aunque la Subsecretaría de Telecomunicaciones va a permitir esta portabilidad, ciertamente el tema relevante ya no se encuentra en la telefonía fija sino que en la telefonía móvil, en donde tres empresas concentran el mercado y el nivel de movilidad de los usuarios de una compañía a otra es prácticamente nulo. Más allá de los servicios regulados, los costos de cambio son comunes en varias industrias y resulta preocupante cómo los programas de fidelización a usuarios en las aerolíneas, farmacias y multitiendas crean poder de mercado que inhibe la entrada de nuevos competidores y extrae rentas en favor de las empresas.

Finalmente, uno de los editores del libro Michel Ghertman ofrece una suerte de síntesis de lo tratado en su artículo "The Puzzle of Regulation, Deregulation and Reregulation" al tratar a la teoría de la regulación, desde Pigou a Stigler, como muy disciplinares y carentes de las visiones alternativas que ofrece la ciencia política. Argumenta Ghertler que la mirada interdisciplinar de los temas regulatorios es sin duda el gran aporte de Oliver Williamson y de la economía de los costos de transacción aplicadas a estos sectores.

Me parece que siendo acertado en buscar una mirada interdisciplinar a un problema netamente interdisciplinar es el camino correcto, aunque ello no invalida los enormes aportes que hicieron a la teoría de la regulación Pigou, Stigler y sus seguidores. No obstante estar de acuerdo con el autor, me hubiese parecido más interesante, por tratarse de miradas contemporáneas, que él hiciera un paralelo entre el enfoque que entrega la nueva economía institucional (que incluye a la economía de los costos de transacción) a estos temas como los aportes de la teoría de los derechos de propiedad aplicada a estos temas regulatorios, en donde la mirada interdisciplinar es también usada, aunque metodológicamente diferente al usar extensivamente las herramientas de teoría de juegos (ver Tirole, 1994). 


\section{EPILOGO}

A modo de resumen y parodiando el título del último artículo del libro, la evolución desde industrias reguladas, sus procesos de liberalización y luego de rerregulación en varios casos no son realmente un puzzle, se explica razonablemente y se justifica esa tendencia en algunos casos mencionados en el propio libro, o al menos se entiende esa tendencia en otros capítulos. Con todo, se trata de un libro que debiera estar en los escritorios de los policy makers y de los académicos aplicados que interactuamos entre la vida universitaria y la realidad de la implementación de políticas en sectores de servicios básicos e infraestructura. Sus enseñanzas sin dudas beneficiarían la calidad de la política regulatoria en nuestros países.

\section{REFERENCIAS}

ARMSTRONG, M., S. COWAN y J. VICKERS (1994). Regulatory Economics. MIT Press.

BAKER, G., R. GIBBONS y K. MURPHY (2002). "Relational Contracts and the Theory of the Firm", Quarterly Journal of Economics 117 (1), pp. 39-84.

BOLTON, P. y M. DEWATRIPONT (2005). Contract Theory. MIT Press.

CREMER, H., M. MARCHAND y J. THISSE (1989). "The Public Firm as an Instrument for Regulating an Oligopolistic Market”, Oxford Economic Papers 41, pp. 283-301.

DE FRAJA, G. y F. DELBONO (1989). "Alternative Strategies of a Public Enterprise in Oligopoly”, Oxford Economic Papers 41, pp. 302-311.

DIXIT, A. (1996). The Making of Economic Policy. Cambridge University Press.

FISCHER-BOLLIN, P. (2008). Eficiencia del Gasto Público en América Latina. Konrad Adenauer Stiftung, Brasil.

HOLMSTRÖM, B. y J. ROBERTS (1998). "The Boundaries of the Firm Revisited”, Journal of Economic Perspectives 12 (4), pp. 73-94.

KAUFMANN, D., A. KRAAY y M. MASTRUZZI (2009). "Governance Matters VII: Aggregate and Individual Governance 1996-2008”, World Bank Policy Research WPS 4978.

LORA, E. (2001), "Structural Reforms in Latin America: What Has Been Reformed and How to Measure It", IADB Working Paper 466.

MENARD, C. (2006). "Hybrid Organization of Production and Distribution", Revista de Análisis Económico 21 (2), pp. 25-41.

NEWBERY, D. (1999). Privatization, Restructuring, and Regulation of Network Utilities. MIT Press.

NORTH, D. (1990). Institutions, Institutional Change, and Economic Performance. Cambridge University Press.

PAREDES, R., J.M. SÁNCHEZ y A. FERNÁNDEZ (1995). "Privatización y Regulación en Latinoamérica", Revista de Análisis Económico 10 (2), pp. 1-20.

TIROLE, J. (1994). “The Internal Organization of Government”, Oxford Economic Papers 46, pp. 1-29. 